ARTÍCULO ESPECIAL

\title{
Sulfonilureas. Estado actual de su empleo en América Latina. Documento de posición de la Asociación Latinoamericana de Diabetes
}

Juan Rosas-Saucedo', Juan Rosas-Guzmán ${ }^{1}$, José Agustín Mesa-Pérez ${ }^{2 *}$, Manuel González-Ortiz ${ }^{3}$, Esperanza Martínez-Abundis ${ }^{3}$, Roberto González-Suárez ${ }^{4}$, Isaac Sinay ${ }^{5}$ y Ruy Lyra ${ }^{6}$

${ }^{1}$ Instituto de Diabetes A.C. Celaya, Guanajuato, México; ${ }^{2}$ Presidente de la Asociación Latinoamericana de Diabetes, Coordinador del Grupo NeuroALAD, Oaxaca de Juárez, México; ${ }^{3}$ Instituto de Terapéutica Experimental y Clínica, Centro Universitario de Ciencias de la Salud, Universidad de Guadalajara, Guadalajara, México; ${ }^{4}$ Socio titular Asociación Latinoamericana de Diabetes; ${ }^{5}$ Asesor Unidad Diabetes del Instituto Cardiovascular de Buenos Aires, Argentina; ${ }^{6}$ Profesor de Endocrinología, Universidad de Federal de Pernambuco, Recife, Brasil

\section{RESUMEN}

El descubrimiento de la acción hipoglucemiante de las sulfonilureas (SU), al igual que ha sucedido con otros fármacos, fue accidental. Se propuso que su mecanismo de acción era mediante la estimulación de la secreción y liberación de insulina por la célula pancreática, sugiriéndose desde entonces que este tipo de fármacos podía ser útil en el tratamiento de diabetes mellitus. Al ser los primeros agentes orales que se desarrollaron para el tratamiento de diabetes mellitus tipo 2 $(\mathrm{DM} 2)$, tienen una larga historia. El tratamiento prolongado con SU ha mostrado disminuir su eficacia con el tiempo, al igual que sucede con casi la totalidad de los fármacos que se utilizan para tratar la DM2. Este fenómeno encuentra explicación en la historia natural de la enfermedad, al existir una pér-

\section{ABSTRACT}

The discovery of the antidiabetic actions of sulphonylureas (SU), just like many other medications, was incidental. The proposed mechanism of action of the SU was through stimulation of the secretion and liberation of insulin by pancreatic cells, suggesting since then that this type of medication could be useful in the treatment of diabetes mellitus. Since SU are the first oral agents developed for type 2 diabetes mellitus, they have a long history of clinical use. The efficacy of the treatment with SU has proven to be reduced over prolonged periods of time, just as almost every other antidiabetic medication used for the treatment of type 2 diabetes. This phenomenon can be explained by the natural history of the disease, the progressive loss of secretory capacity by the beta-cell, seen even with the use of insulin. The clinical experience 
dida progresiva de la capacidad secretora por parte de la célula beta, y se presenta incluso con el uso de insulina. La experiencia de uso y los resultados de los diferentes estudios que incluyen a las SU no han mostrado incremento en la mortalidad en los pacientes que las utilizan e incluso se ha encontrado reducción de la mortalidad global asociada al control de los demás factores de riesgo. El empleo de las SU está respaldado en todas las guías y algoritmos internacionales para el tratamiento de la DM2, lo que las convierte, dado su bajo costo, potencia, facilidad de administración, potencial de combinación con otros medicamentos y seguridad, en medicamentos a tomar en cuenta al decidir el tratamiento más adecuado.

Palabras clave: Diabetes. Sulfonilureas. Seguridad. Tratamiento. with its use, and the results of the different studies including SU have not shown an increase in mortality of the patients who regularly use them, and even a reduction in overall mortality was found when there was an adequate control of the rest of risk factors. Use of SU is backed by every international clinical guideline and algorithm of type 2 diabetes, which makes them, given their low cost, potency, ease of use, combination potential with other antidiabetics and safety, a medication to consider when deciding the most appropriate treatment. (Rev ALAD. 2019;9:92-106) Corresponding author: José Agustín Mesa-Pérez, presidenciaalad1619@gmail.com

Key words: Diabetes. Sulphonylurea. Safety. Treatment.

\section{INTRODUCCIÓN}

Al igual que ha sucedido con otros fármacos, el descubrimiento de la acción hipoglucemiante de las sulfonilureas (SU) fue accidental. En 1942, Janbon, et al., en la clínica de enfermedades infecciosas de la Facultad de Medicina de Montpellier, al estudiarse la sulfamida RP 2254 en busca de alternativas para el tratamiento de la fiebre tifoidea, observaron la aparición de crisis convulsivas en algunos de sus pacientes. Auguste Loubatières observó que las convulsiones observadas eran similares a las que él obtenía al inducir hipoglucemias con altas dosis de insulina. La hipótesis de la inducción de los efectos hipoglucemiantes a partir de la sulfamida RP 2254 fue comprobada en perros y ratas con diabetes por aloxano, en los que además se observó que la administración del producto reducía los niveles plasmáticos de glucosa1. Las primeras pruebas en seres humanos mostraron que la RP 2254 reducía los niveles de glucosa en personas con diabetes.

Estos y otros estudios llevaron a Loubatières a proponer que el mecanismo de acción de esta SU se obtenía mediante la estimulación de la secreción y liberación de insulina por la célula pancreática, sugiriéndose desde entonces que este tipo de fármacos podía ser útil en el tratamiento de la diabetes mellitus, dado su poder reductor sobre la glucemia'.

Las SU fueron los primeros agentes orales que se desarrollaron para el tratamiento de la diabetes mellitus tipo 2 (DM2) en la década de los años 50 y tienen 
una larga historia de uso clínico. Desde que las primeras SU estuvieron disponibles, esta clase de fármacos ha pasado por varias etapas de desarrollo y, por lo tanto, los medicamentos que la integran presentan diferentes propiedades farmacológicas².

En el desarrollo del presente documento mostraremos los resultados de los principales estudios de investigación con los beneficios del uso de SU, como los reportados en el United Kingdom Prospective Diabetes Study (UKPDS) ${ }^{3,4}$, que mostró que las SU en el brazo intensivo redujeron de 1 a $1.5 \%$ los niveles de hemoglobina glucosilada A1c (HbA1c) en los dos primeros años de tratamiento en pacientes recién diagnosticados con DM2 y donde disminuyeron hasta un 9\% la mortalidad total, un $22 \%$ el infarto miocardio y un $43 \%$ las complicaciones microvasculares, y se observó un incremento de $1.7 \mathrm{~kg}$ promedio, con un 1.4\% de episodios de hipoglucemias por año.

El tratamiento prolongado con SU ha mostrado disminuir su eficacia con el tiempo, al igual que sucede con casi la totalidad de los fármacos que se utilizan para tratar la DM2. Este fenómeno, conocido como fracaso secundario a las SU, tiene lugar entre un 3 y un 10\% de los pacientes por año. Este fenómeno encuentra explicación en la historia natural de la DM2 y su patogenia, al existir una pérdida progresiva de la capacidad secretora por parte de la célula beta y se presenta incluso con el uso de insulina.

Ni el UKPDS, ni el Diabetes Mellitus Insulin-Glucose Infusion in Acute Myocardial Infarction (DIGAMI) $2^{5}$, ni el Action in Diabetes and Vascular Disease: Preterax and Diamicron MR Controlled Evaluation (ADVANCE) ${ }^{6}$ mostraron incremento de la mortalidad entre pacientes que utilizaban SU.

Otros estudios también muestran el actuar de las SU: A Diabetes Outcome Progression Trial (ADOPT) 7,8 , que es referente para evaluar la durabilidad del tratamiento de fármacos orales e incluye SU, y el estudio ADVANCE, que nos mostró eficacia, seguridad y da base a un efecto legado, visto después de varios años de su manejo. También el estudio Steno- $2^{9}$ mostró resultados favorables en la reducción de la mortalidad global con uso de $\mathrm{SU}$ en un contexto de control multifactorial de factores de riesgo, ya que entre el 30 y el 50\% de los pacientes con DM2 utilizan estos fármacos como parte de su tratamiento habitual.

El empleo de las SU está respaldado en todas las guías y algoritmos internacionales para el tratamiento de la DM2, lo que las convierte, dado su bajo costo, potencia, facilidad de administración, potencial de combinación con otros medicamentos y su seguridad, en medicamentos a tomar en cuenta en el momento de decidir el tratamiento más adecuado.

\section{ASPECTOS FARMACOLÓGICOS RELEVANTES DE LAS SULFONILUREAS}

Las SU estimulan la secreción de insulina de una forma no dependiente de la glucosa, de acuerdo a estudios in vitro, y dependiente de la glucosa a concentraciones terapéuticas, en estudios in vivo; lo hacen al unirse a receptores específicos en la membrana plasmática de las células beta pancreáticas, lo que resulta en el cierre de los canales de potasio sensibles al trifosfato de adenosina (KATP), lo cual desencadena la despolarización celular con la apertura de los canales de calcio dependientes de voltaje e incremento del calcio intracelular y la exocitosis de los gránulos que contienen insulina ${ }^{10}$ (Fig. 1). El efecto insulinotrópico aumenta en presencia de hiperglucemia y las nuevas SU aparentemente mejoran ambas fases de la secreción de insulina. Por otra parte, no hay evidencia de que las SU aumenten la síntesis de insulina. 


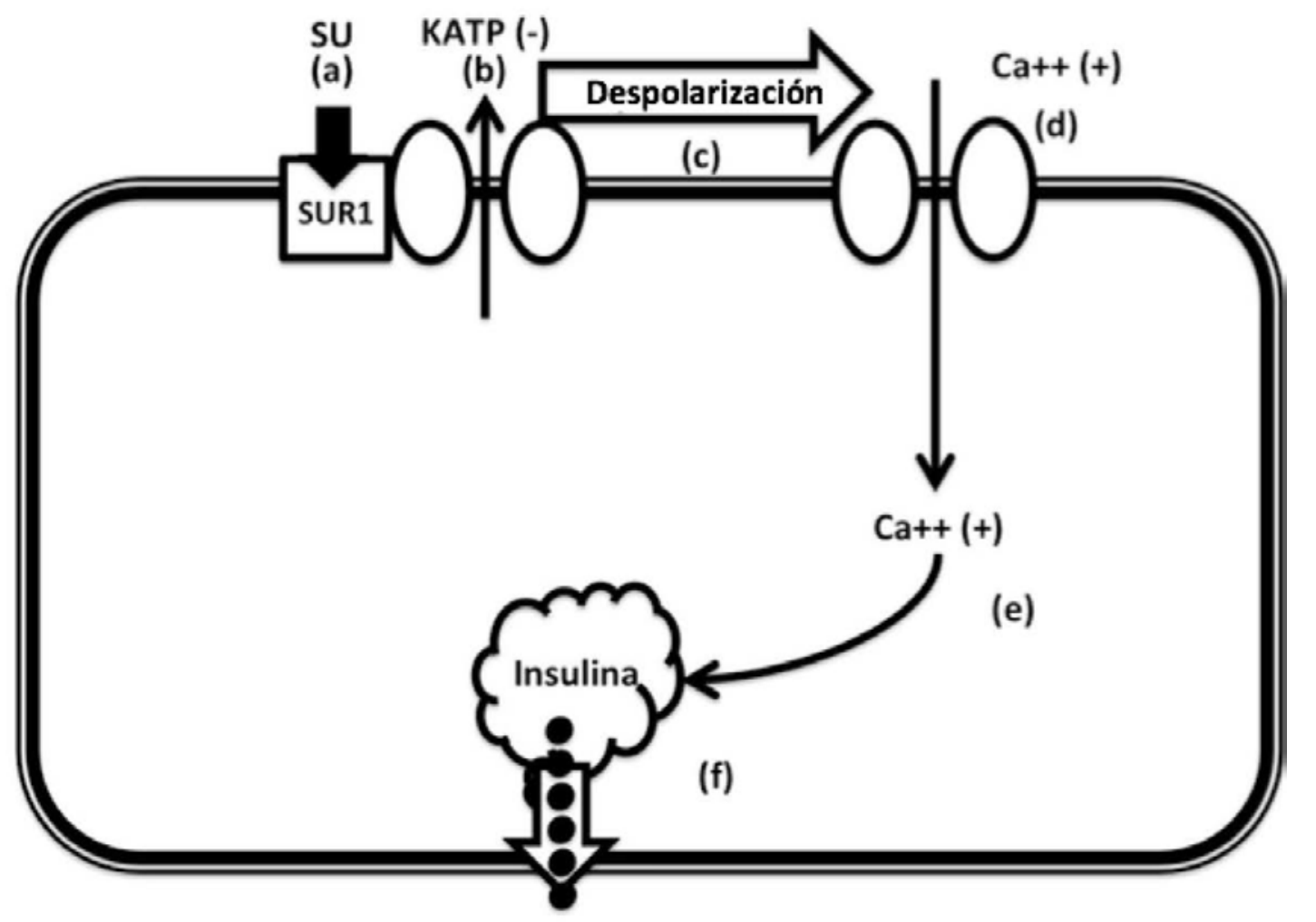

Figura 1. Secreción de insulina: la SU se une al SUR1 (a), se inhibe la KATP (b), se depolariza la célula B pancreática (c), se estimula el transporte de $\mathrm{Ca++}$ (d), se transloca el Ca++ (e) y se estimulan los microtúbulos de insulina y se produce la exocitosis de los gránulos con insulina (f) (adaptada de Kalra, et al., 201810).

Los canales KATP son un complejo constituido por dos proteínas: una subunidad de unión a fármacos (SUR) de la que se han identificado cuatro subtipos, que funcionan como receptores de SU, y una subunidad para la formación de poros y rectificación de la entrada de $\mathrm{K}$ (Kir) con cuatro subtipos, Kir6.2 para células beta del pancreáticas y músculo cardíaco y Kir6.1.15 para músculo liso vascular ${ }^{11}$.

Se han identificado dos genes para los receptores de SU, los cuales codifican a las proteínas SUR1 y SUR2. El predominio del tipo de SUR varía entre los tejidos: el SUR1 se encuentra predominantemente en las células beta pancreáticas (receptores de alta afinidad) y en el cerebro, el SUR2A en músculo cardíaco y esquelético y el SUR2B en músculo liso vascular ${ }^{11}$ (receptores de baja afinidad) (Fig. 2).
Se ha considerado que la apertura de los canales KATP cardíacos protege al corazón durante los periodos de isquemia. Las SU con afinidad por los SUR2A pueden inhibir su apertura, mientras que los que actúan selectivamente en los receptores SUR1 en las células beta pancreáticas pueden presentar un menor riesgo cardiovascular, por lo que serían las preferidas en personas con alto riesgo de isquemia miocárdica; tal es el caso de la glimepirida y la gliclazida, que no afectan al preacondicionamiento isquémico del corazón, al regular la vasodilatación como un mecanismo de protección endógeno durante la isquemia y reperfusión miocárdica ${ }^{11}$.

Por otra parte, ciertas SU pueden aumentar directamente la generación de especies reactivas de oxígeno y potencialmente causar apoptosis de las 
A

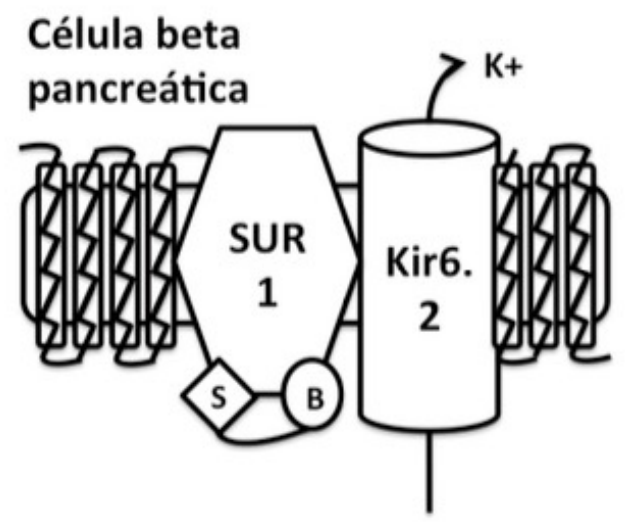

B

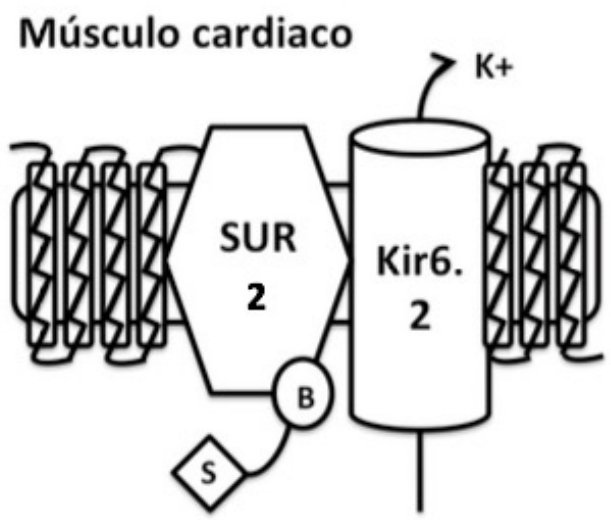

FIgURA 2. A: complejo transmembrana del receptor SUR1 y el flujo de salida del potasio Kir6.2 en la células beta pancreáticas con el sitio de unión citosólico a SU (S) y el sitio de unión benzamida (B). B: receptor SUR2A o SUR2B sin sitio de unión a SU (adaptada de Colagiuri, et al., 201811).

células beta pancreáticas. Se sabe que la gliclazida depura radicales libres por medio del anillo azabiciclo-octilo del núcleo de las SU, por lo que pudiera proteger a las células beta pancreáticas de la apoptosis inducida por el estrés oxidativo y clínicamente manifestarse por un menor fracaso al tratamiento en comparación con la glibenclamida y la glipizida ${ }^{11}$.

Además, la gliclazida es la única SU que no se une al receptor Epac2, el cual es un estimulante de la exocitosis de la insulina y que aunado con la rápida reversibilidad de su asociación al SUR1 produce una menor sobreestimulación pancreática, lo que permite una recuperación de la falla de la primera fase de secreción de insulina, con un menor riesgo en la aparición de hipoglucemia. De igual manera, la glimepirida ocasiona menor hipoglucemia y aumento de peso en comparación con las SU de primera generación, debido a una menor afinidad y rápida asociación-disociación al receptor ${ }^{11}$.

Como se observa en la tabla 1, la vida media de las SU puede variar desde una hora hasta dos días y la duración de su efecto desde seis horas hasta tres
TABLA 1. Vida media y duración del efecto de las sulfonilureas

\begin{tabular}{|l|c|c|}
\hline & \multicolumn{1}{|c|}{$\begin{array}{c}\text { Vida media, } \\
\text { horas }\end{array}$} & $\begin{array}{c}\text { Duración del } \\
\text { efecto, horas }\end{array}$ \\
\hline Primera generación & $3-28$ & $6-12$ \\
\hline Tolbutamida & $28-48$ & $24-72$ \\
\hline Clorpropamida & $2-4$ & $12-24$ \\
\hline Glibenclamida & $1-5$ & $12-18$ \\
\hline Glipizida & \multicolumn{2}{|c|}{} \\
\hline $\begin{array}{l}\text { Nueva generación } \\
\text { Glimepirida }\end{array}$ & $5-9$ & 24 \\
\hline $\begin{array}{l}\text { Gliclazida } \\
\text { modiberación }\end{array}$ & $10-12$ & $16-11$ \\
\hline \begin{tabular}{l} 
Gliclazida de \\
\hline
\end{tabular} & & \\
\hline
\end{tabular}

días, lo que hace que las de primera generación como la tolbutamida deban de prescribirse varias veces al día, con lo que disminuye el apego al tratamiento, y que las otras, por su efecto prolongado, aumenten el riesgo de hipoglucemia. Las de nueva generación por sus características son más recomendadas. 
Finalmente, las SU de más reciente generación presentan diversos efectos farmacológicos, no necesariamente relacionados con la secreción de insulina (efecto pleiotrópico), pero muy favorables para el control óptimo del paciente con DM2 como serían: a) estimulación de la secreción de somatostatina por las células alfa pancreáticas, b) disminución de la hormona del crecimiento, c) depuración hepática de insulina, d) reducción en la secreción de glucagón, e) disminución de la producción hepática de glucosa, f) sensibilización periférica a la insulina en músculo, adipocitos e hígado, g) efecto antioxidante, h) aumento de adiponectina, i) estimulación de la lipogénesis, j) disminución del colesterol total y triglicéridos, k) aumento en las concentraciones del colesterol de las lipoproteínas de alta densidad, I) disminución en marcadores de inflamación (proteína $C$ reactiva de alta sensibilidad y factor de necrosis tumoral alfa) y de disfunción endotelial (molécula 1 de adhesión a las células vasculares, molécula de adhesión intercelular), m) reducción de la hipercoagulabilidad, n) aumento de la fibrinólisis, ñ) disminución de la agregación plaquetaria, o) disminución de angiogénesis, y p) mejoría de la salud vascular y del preacondicionamiento isquémico ${ }^{10}$.

\section{EFICACIA TERAPÉUTICA DE LAS SULFONILUREAS EN EL CONTROL DE LA GLUCEMIA Y OTROS EFECTOS METABÓLICOS RELEVANTES}

Los agentes que pertenecen a esta clase presentan eficacia similar para reducir la HbA1c, sin embargo, difieren en términos de frecuencia y magnitud de hipoglucemia, ganancia de peso, seguridad cardiovascular y renal. Pueden ser empleadas como monoterapia y terapia combinada doble o triple, prácticamente con cualquier otro agente antidiabético oral o inyectable, incluso insulina, a excepción de las glinidas ${ }^{10}$.

\begin{tabular}{|c|c|c|}
\hline Sulfonilureas & Dosis diaria, mg & $\begin{array}{l}\text { Titulación } \\
\text { de dosis }\end{array}$ \\
\hline \multicolumn{3}{|c|}{ Primera generación } \\
\hline Tolbutamida & $1,000-3,000$ & \\
\hline Clorpropamida & $100-500$ & \\
\hline Glibenclamida & $1.25-20$ & $\begin{array}{l}\text { No }>2.5 \text { mg/día } \\
\text { cada semana }\end{array}$ \\
\hline Glipizida & $5-40$ & $\begin{array}{l}2.5-5 \mathrm{mg} / \mathrm{dí} \text { cada } \\
\text { 1-2 semanas }\end{array}$ \\
\hline \multicolumn{3}{|c|}{ Nueva generación } \\
\hline Glimepirida & $1-8$ & $\begin{array}{l}\text { 1-2 mg/día cada } \\
\text { 1-2 semanas }\end{array}$ \\
\hline Gliclazida & $40-320$ & $\begin{array}{l}40 \text { mg/día cada } \\
2-4 \text { semanas }\end{array}$ \\
\hline $\begin{array}{l}\text { Gliclazida de } \\
\text { liberación } \\
\text { modificada }\end{array}$ & $30-120$ & $\begin{array}{l}\text { 30-60 mg/día cada } \\
\text { 2-4 semanas }\end{array}$ \\
\hline
\end{tabular}

Ensayos clínicos controlados, metaanálisis y revisiones sistemáticas con SU han mostrado su alta eficacia para reducir la $\mathrm{HbA} 1 \mathrm{c}$ en corto tiempo y en varios estudios constituyen el estándar de comparación con otros tratamientos para DM2. En la tabla 2 se pueden consultar las dosis diarias y su titulación; se recomienda iniciar con dosis bajas y titular gradualmente con base en la respuesta glucémica para prevenir la hipoglucemia. Asimismo, se deberán considerar otros factores con el empleo de SU, como la actividad física, la omisión de alimentos, y la presencia de vómitos y diarreas, entre otros ${ }^{10,12}$.

El estudio GRADE'13 (Enfoques de reducción de la glucemia en la diabetes: un estudio comparativo de efectividad), que se encuentra en curso, comparará el control glucémico con cuatro clases de fármacos (SU, inhibidores de la dipeptidil-peptidasa 4 [iDPP4], agonistas del receptor del péptido similar al glucagón-1 e insulina basal) agregados a metformina, durante 4 años, en pacientes con DM2 de reciente inicio. 
Con relación a la durabilidad del efecto de las SU, el estudio ADOPT, que incluyó 4,360 pacientes, mostró que la metformina, la glibenclamida y la rosiglitazona empleadas como monoterapia inicial para el tratamiento de la DM2 lograron un control glucémico $(H b A 1 c \leq 7 \%)$ similar hasta durante 2.75 años, con inicio de la divergencia alrededor de los 3 años a favor de la rosiglitazona con una mayor durabilidad. La incidencia acumulada de falla secundaria fue del $21 \%$ con metformina, $34 \%$ con glibenclamida y $15 \%$ con rosiglitazona a 5 años y la glibenclamida se asoció con un menor riesgo de eventos cardiovasculares (incluida la insuficiencia cardíaca congestiva) que la rosiglitazona ( $p<0.05)$, y el riesgo asociado con la metformina fue similar al de la rosiglitazona en ese estudio ${ }^{14}$. El estudio ADVANCE, con 11,140 pacientes con $\mathrm{DM}_{2}$, mostró que un control intensivo de glucosa $(\mathrm{HbA} 1 \mathrm{c} \leq 6.5 \%)$ con el uso de gliclazida (liberación modificada) y otros medicamentos redujo de manera significativa la nefropatía (21\%) y el beneficio persistió en un seguimiento a 9.9 años, sin evidencia de un mayor riesgo de eventos cardiovasculares o muerte ${ }^{6,15}$.

El estudio CAROLINA ${ }^{16}$ (diseño y características basales del ensayo de resultados cardiovasculares de linagliptina vs. glimepirida en DM2), que culminará en 2019, comparará una SU (glimepirida) y un iDPP4 (linagliptina) y proporcionará datos de resultados cardiovasculares. El estudio evaluará como desenlaces primarios los eventos cardiovasculares mayores de 3 puntos: mortalidad cardiovascular, infarto del miocardio no fatal y enfermedad vascular cerebral no fatal, así como otros desenlaces secundarios, y será el primer estudio que evalúe la seguridad cardiovascular de una SU con el diseño propuesto por la Food and Drug Administration para este fin.

Algunas de las consideraciones para utilizar las diferentes SU son: la glibenclamida debe limitarse a aquellos pacientes con tasa de filtración glomerular $($ TFG) $>60 \mathrm{ml} / \mathrm{min}$; la glimepirida se puede emplear con una TFG > $60 \mathrm{ml} / \mathrm{min}$ y con dosis reducida en TFG de 30-60 ml/min; la gliclazida y la glipizida tienen metabolitos inactivos y el riesgo de hipoglucemia en insuficiencia renal es menor y se pueden usar con valores de TFG > $30 \mathrm{ml} / \mathrm{min}$ y en dosis reducidas por debajo de este nivel con un monitoreo cuidadoso. Se recomienda reducción de las dosis de SU o aumento en su intervalo de dosificación en pacientes con insuficiencia hepática leve y moderada. Deben ser utilizadas con precaución en el paciente adulto mayor ${ }^{10,11}$.

Las SU cuentan con presentaciones de combinación a dosis fijas con metformina y otros fármacos, lo que incrementa la adherencia al tratamiento, mejora los resultados clínicos y la satisfacción del paciente, y reduce los $\operatorname{costos}^{10}$.

Nuevas generaciones de SU parecen producir menos hipoglucemias y aumento de peso. La favorable relación de costo y eficacia de las SU las hace asequibles para la mayoría de las economías en atención para la salud².

\section{RIESGO DE HIPOGLUCEMIAS CON EL USO DE SULFONILUREAS}

El principal efecto adverso del tratamiento con SU es la hipoglucemia. Los síntomas comúnmente asociados a hipoglucemia son secundarios a la disminución de la función neuronal y la secreción de hormonas contrarreguladoras como el glucagón y la adrenalina. En sujetos normales, la secreción de insulina cesa alrededor de los $81 \mathrm{mg} / \mathrm{dl}$ de glucemia; si este nivel disminuye por debajo de los $68 \mathrm{mg} / \mathrm{dl}$, se secretan hormonas contrarreguladoras y son causa de síntomas autonómicos como sudoración, temblor e irritabilidad. Al llegar a los $50 \mathrm{mg} / \mathrm{dl}$ pueden aparecer síntomas 
TABLA 3. Clasificación de hipoglucemia

\begin{tabular}{|l|l|}
\hline Hipoglucemia grave & $\begin{array}{l}\text { Requiere la ayuda de otra persona para la administración de carbohidratos, glucagón o } \\
\text { medidas de resucitación }\end{array}$ \\
\hline Hipoglucemia clínicamente relevante & Documentación de glucosa $\leq 54 \mathrm{mg} / \mathrm{dl}$, independientemente de los síntomas presentados \\
\hline Hipoglucemia sintomática documentada & Síntomas típicos de hipoglucemia con una medición de glucosa $\leq 70 \mathrm{mg} / \mathrm{dl}$ \\
\hline Hipoglucemia asintomática & Documentación de glucosa $\leq 70 \mathrm{mg} / \mathrm{dl}$ que no se acompaña de síntomas típicos \\
\hline Hipoglucemia sintomática probable & Síntomas típicos de hipoglucemia sin medición de glucosa \\
\hline Hipoglucemia relativa & $\begin{array}{l}\text { Síntomas sugerentes de hipoglucemia, el paciente los identifica como tales, pero la medición } \\
\text { de glucosa es }>70 \mathrm{mg} / \mathrm{dl}\end{array}$ \\
\hline
\end{tabular}

neuroglucopénicos importantes, como alteraciones cognitivas, confusión e incluso convulsiones. Debajo de este nivel, puede aparecer el coma, posteriormente el aplanamiento de las ondas electroencefalográficas y muerte neuronal por abajo de los $18 \mathrm{mg} / \mathrm{dl}$. La exposición recurrente a hipoglucemia puede alterar de forma crónica la respuesta contrarreguladora a la hipoglucemia y por tanto la alerta antes de llegar a presentar datos de neuroglucopenia ${ }^{17}$.

Son los eventos graves de hipoglucemia los que causan la mayor ansiedad a los pacientes y sus médicos y tienen implicaciones importantes en la vida diaria del paciente, así como su bienestar. Los eventos de hipoglucemia se clasifican como se describe en la tabla 3.

En estudios observacionales, se ha reportado que la incidencia general de hipoglucemia con el uso de SU puede ser de alrededor del 1.8\% al año. En un análisis de estos estudios, condiciones como edad avanzada y el uso concomitante de medicamentos hipoglucemiantes se asocian a una mayor incidencia de hipoglucemias. El uso de SU solo de forma ocasional o cambiar constantemente de tratamiento también conlleva un riesgo incrementado de eventos adversos, se llega al menos a triplicar la cantidad de hipoglucemias documentadas, si se compara con pacientes que son usuarios de forma continua de sus medicamentos ${ }^{18}$.
Una de las complicaciones frecuentes en los pacientes con diabetes es el deterioro progresivo de la función renal. Uno de los grandes puntos a considerar en los pacientes con disminución de la TFG es la eliminación o ajuste de los diversos medicamentos nefrotóxicos o con eliminación renal que se utilizan. En este grupo de pacientes, los eventos de hipoglucemia reportados tienen una gran variabilidad, pueden llegar a ser tan altos como el 33\% por paciente por año con al menos una hipoglucemia sintomática.

En este sentido, si se toman en cuenta las propiedades farmacológicas de las SU, podemos observar dos grupos, aquellas de vida media larga con metabolitos activos eliminados por vía renal, como la glibenclamida y la glimepirida, y las de vida media más corta y con metabolitos inactivos o excretadas como molécula intacta, como la gliclazida, la glipizida y la tolbutamida, que ya poseen un mínimo efecto hipoglucemiante al momento de ser eliminadas. De forma paralela al declive de la función renal, se tendrá un acúmulo de estos metabolitos y aquellos con propiedades activas residuales tendrán una mayor incidencia de eventos de hipoglucemia ${ }^{19}$.

La dosis del medicamento es otro factor para tomar en cuenta. En aquellos pacientes que utilizan la dosis máxima de SU se ha documentado riesgo de hasta 3.6 veces más de hipoglucemia comparado con el uso de metformina sola. El riesgo va de la 
mano con la función renal, se tiene un incremento de riesgo de hasta 5 veces en los pacientes con una TFG estimada menor a $30 \mathrm{ml} / \mathrm{min}$. Cuando se compara por compuestos individuales, la glibenclamida fue la de mayor riesgo de hipoglucemia, llegando a 7.5 veces mayor comparada con metformina. Otros compuestos, como gliclazida, glimepirida, glipizida y tolbutamida tuvieron un menor riesgo, que osciló entre 1.5 y 2.5 , que es similar entre estos últimos fármacos ${ }^{19}$.

Los pacientes con diabetes suelen tener prescripción de múltiples fármacos para enfermedades asociadas. Las interacciones farmacológicas que tienen pueden potenciar el riesgo de hipoglucemia mediante la inhibición de las enzimas del citocromo P450 hepático (CYP) implicadas en su metabolismo. Específicamente, los tratamientos para la hipertrigliceridemia frecuentemente utilizados pueden inhibir la CYP3A y la CYP2C9, ambas responsables de la inhibición de la glibenclamida, y la última de la glipizida y la glimepirida. Estas interacciones pueden resultar en incremento en los efectos hipoglucemiantes de las SU y el riesgo de hipoglucemias e hipoglucemias graves ${ }^{20}$.

El uso concomitante de SU y fenofibrato se asoció con un incremento del $20-60 \%$ en la tasa de hipoglucemias graves y hasta 2.5 veces más frecuente con el uso de glimepirida alrededor del tercer y cuarto mes de uso; esto al aumentar las concentraciones plasmáticas de la SU hasta en un $23 \%$. En cuanto a la asociación con gemfibrozil, aumentó en 40-60\% las hipoglucemias graves sobre todo al prescribirse en conjunto con glipizida, donde el riesgo aumentó hasta 2.4 veces alrededor del quinto y sexto mes de uso. Se han propuesto otras vías causales como la estimulación de los receptores activados de proliferación de los peroxisomas a por los fibratos o la inhibición de polipéptidos transportadores de aniones orgánicos 20 .
En caso de estar ante una disminución de la glucosa por debajo de los $70 \mathrm{mg} / \mathrm{dl}$, se deberá corregir a la brevedad. Si el paciente se encuentra consciente y es capaz de deglutir de forma segura, se deberá preferir la vía oral para la administración de glucosa; solo en caso de disminución del estado de alerta, donde el paciente no pueda usar la vía oral de forma segura, se considerará el tratamiento parenteral. Como guía general, si el paciente presenta una hipoglucemia sintomática, será necesario ingerir carbohidratos de la fuente más cercana disponible; entre los alimentos más utilizados se encuentran las frutas, jugos, refrescos o caramelos. En caso de estar ante una hipoglucemia asintomática, será suficiente con adelantar el tiempo de comida siguiente o en su defecto tomar una cantidad de carbohidrato menor a la requerida en un episodio sintomático. En caso de tener disponible glucagón, este deberá administrarse, $1 \mathrm{mg}$ para pacientes de más de $20 \mathrm{~kg} 00.5 \mathrm{mg}$ en menores de $20 \mathrm{~kg}$, por inyección subcutánea, intramuscular o intravenosa, pudiendo repetir una o dos dosis extras, sin que esto retrase la administración de glucosa en caso de hipoglucemia grave ${ }^{21}$.

La vida media larga de la mayor parte de las SU supone la necesidad de vigilancia clínica y con glucemia capilar más prolongada después de un episodio de hipoglucemia. Generalmente, será necesario descartar nuevos episodios de hipoglucemia en las 24 a 48 horas siguientes a la última dosis de SU, incluso hasta las 72 horas en pacientes ancianos o con insuficiencia renal. En algunos casos es necesario mantener al paciente en hospitalización y con infusión de glucosa hasta lograr la euglucemiaํ․

La hipoglucemia grave tiene un efecto profundo y potencialmente irreversible en el cerebro. A nivel celular la falta de energía puede llevar a interrupciones de actividades celulares críticas. Desde el punto de vista clínico, esto se manifiesta como disfunción cognitiva progresiva de manera paralela al 
número y gravedad de las hipoglucemias de los pacientes. Es por ello que será de vital importancia minimizar los riesgos de padecerlas, lograr identificarlas de forma temprana y resolverlas de forma inmediata para evitar secuelas a largo plazo.

\section{¿HAY RIESGO CARDIOVASCULAR CON EL USO DE SULFONILUREAS?}

La seguridad cardiovascular de las SU es una cuestión de debate. Las posibles razones para un incremento de riesgo cardiovascular serían que las SU están asociadas al aumento de la proinsulina circulante, que puede elevar los niveles de inhibidor del activador del plasminógeno 1, el principal inhibidor fisiológico de la fibrinólisis, con lo que se aumentaría así el riesgo de aterotrombosis ${ }^{22}$. Otra hipótesis sugiere que las SU pueden inhibir el preacondicionamiento isquémico ${ }^{10}$, un mecanismo cardioprotector en el que periodos cortos de isquemia miocárdica pueden reducir el tamaño de un infarto futuro. Las SU bloquean los canales KATP en las células beta, pero también se unen e inhiben selectivamente los canales KATP en los cardiomiocitos y en las células musculares lisas vasculares. Esto, a su vez, lleva a la hiperpolarización de la membrana y al acortamiento del potencial de acción cardíaca, con reducción de la contractilidad y con mayor perjuicio sobre los mecanismos de protección contra la isquemia miocárdica. Algunas evidencias sugieren que la gliclazida y la glimepirida no perjudican el preacondicionamiento isquémico por su escasa interacción con el receptor SUR2. El aumento de la hipoglucemia con el uso de SU se considera un contribuyente indirecto para el riesgo cardiovascular. Los estudios recientes han demostrado que la hipoglucemia está asociada a niveles aumentados de marcadores proinflamatorios y proaterotrombóticos, disminución de respuestas endoteliales arteriales y aumento de la dinámica del QT con prolongación del QTC. La ganancia de peso es otro efecto adverso comúnmente reportado del tratamiento con SU, lo que podría considerarse un factor de riesgo cardiovascular establecido.

En cuanto a estudios que evalúan el uso de SU y el riesgo cardiovascular, muchos de estos ensayos analizaron diferentes niveles de control glucémico como la principal intervención para disminuir la enfermedad cardiovascular, y debido a que los grupos de abordaje intensivo en esos estudios tuvieron una mayor exposición a SU, estos estudios también proporcionan evidencia indirecta sobre la seguridad de estos fármacos. La mayoría de estos estudios (Veterans Affairs Diabetes Trial [VADT], ADVANCE, Action to Control Cardiovascular Risk in Diabetes [ACCORD] y UKPDS) demostraron una disminución en las complicaciones microvasculares, pero ninguno de ellos demostró una reducción inequívoca de los resultados macrovasculares ${ }^{22}$. La única excepción en el seguimiento a largo plazo fue el UKPDS, que mostró que los pacientes que fueron originalmente asignados aleatoriamente a recibir tratamiento intensivo mostraron una reducción estadísticamente significativa en el infarto de miocardio en comparación con los asignados a tratamiento conservador, incluso en aquellos en uso de SU o insulina. Este estudio proporciona, por lo menos, evidencia indirecta de que estos agentes son seguros, y tal vez incluso beneficiosos por la mejora de la glucemia y posible alcance de las metas glucémicas. El estudio ADVANCE también comparó un objetivo estándar de glucosa a un enfoque glucémico intensivo en pacientes con alto riesgo cardiovascular en oposición a los recién diagnosticados con DM2 en el UKPDS. Después de un periodo de seguimiento de 5 años, ocurrieron menos eventos macrovasculares en el grupo de control intensivo, pero la diferencia entre los grupos no alcanzó significancia estadística. Los resultados de este estudio solo proporcionan evidencias circunstanciales para la seguridad de la SU, ya que el estudio evaluó principalmente el efecto del control glucémico, en lugar del perfil de seguridad de las SU. 
No hay un ensayo definitivo de desenlace cardiovascular que evalúe específicamente la seguridad de las SU vs. placebo u otras clases de hipoglucemiantes orales, y por lo tanto la controversia y el debate sobre este tema continúan. La falta de evidencias inequívocas directas sobre la seguridad cardiovascular de estos agentes alimentó una infinidad de relatos que solo tratan de proporcionar algunas respuestas a esta cuestión clínicamente relevante. Todos son estudios observacionales o revisiones sistemáticas y metaanálisis de estudios menores, todos tienen sesgos y limitaciones inherentes que limitan una conclusión definitiva. Por lo tanto, no es sorprendente que sus resultados sean conflictivos, algunos sugiriendo la presencia de un riesgo cardiovascular excesivo con el uso de SU, mientras que otros no encuentran ese riesgo. Los estudios retrospectivos son susceptibles a errores de clasificación de exposición, sesgo de intervalo de tiempo y sesgo de selección. Los estudios clínicos aleatorizados controlados utilizados para los metaanálisis carecen de una evaluación sistemática de los resultados de interés, tienen un periodo de seguimiento muy corto y representan una población heterogénea con muchos factores de confusión y controles que también pueden influir en el riesgo cardiovascular. Rados, et al. realizaron un metaanálisis de 47 ensayos clínicos controlados, todos con una duración superior a 52 semanas, con un total de 37,650 pacientes, para explorar aún más la seguridad cardiovascular de las SU de segunda y tercera generación ${ }^{23}$. En estos estudios, estas SU no se asociaron con un riesgo aumentado de ninguno de los principales resultados evaluados: muerte por todas las causas (OR: 1.12; IC 95\%: 0.96-1.30), muerte cardiovascular (OR: 1.12; IC 95\%: 0.87-1.42), infarto de miocardio (OR: 0.92; IC 95\%: 0.76-1.12) o accidente cerebrovascular (OR: 1.16; IC 95\%: 0.81-1.66) ${ }^{10}$. Estos resultados fueron similares cuando los brazos de SU se compararon con las intervenciones placebo/ dieta o se compararon con otras intervenciones $\operatorname{activas}^{24}$. Por último, las SU analizadas individualmente y tal vez la dosis de exposición también pueden estar asociadas a un riesgo cardiovascular diferenciado. Una revisión de Cochrane de 72 ensayos clínicos aleatorizados que contenían 22,589 individuos aleatorizados para SU u otras intervenciones de control con un periodo de intervención que varió de 24 semanas a 10.7 años reveló que de todas las $\mathrm{SU}$, la gliclazida está asociada a un menor riesgo de eventos cardiovasculares y mortalidad, comparable al de la metformina. Este análisis también relató que el uso de SU de primera generación, como la glibenclamida, comparado al placebo, mostró un aumento estadísticamente significativo del riesgo de muerte cardiovascular (riesgo relativo [RR] 2.63; IC 95\%: 1.32-5.22; $p=0.006$ ). Los posibles efectos cardiovasculares dispares de varias SU también son apoyados por un reciente estudio retrospectivo que contiene 11,141 individuos con DM2. Este estudio no mostró aumento en la mortalidad general con el uso de glipizida, glibenclamida y glimepirida, pero notó un aumento general no significativo en la mortalidad en pacientes con enfermedad arterial coronaria preexistente tratados con glibenclamida vs. glimepirida (Hazard Ratio [HR]: 1.36; IC 95\%: 0.961.91) y glipizida vs. glimepirida (HR: 1.39; IC 95\%: 0.99-1.96), lo que sugiere que la glimepirida puede ser una alternativa mejor cuando se utiliza SU en esa población. Estos datos colectivamente orientan a que las SU de última generación posiblemente presenten un perfil de mayor seguridad cardiovascular, cuando son comparadas con las SU de la primera generación ${ }^{25}$.

\section{OTROS RIESGOS Y EFECTOS ADVERSOS}

Los principales efectos adversos graves asociados con el tratamiento de la DM2 con SU son el aumento del riesgo de hipoglucemia. 
Pueden ocurrir otros efectos adversos de menor gravedad, como trastornos hematológicos, cutáneos y gastrointestinales, en el 2 al $5 \%$ de los pacientes tratados.

Un gran número de ensayos clínicos experimentales, observacionales y metaanálisis han mostrado que los pacientes con DM2 presentan una incidencia mayor de algunos tipos de cáncer, principalmente el de páncreas y el de hígado. Se han propuesto algunos mecanismos para explicar este fenómeno, como el estímulo del crecimiento celular por la hiperglucemia, la resistencia a la insulina, el hiperinsulinismo y el aumento de los niveles circulantes de factores de crecimiento y de citocinas inflamatorias. Igualmente, se ha reportado que numerosos fármacos en uso en el tratamiento de la diabetes tienen algún efecto sobre el riesgo de los pacientes de padecer cáncer debido a su capacidad para influir en algunos de los anteriores factores patogénicos.

En los últimos diez años se han publicado un gran número de ensayos clínicos sobre este tema y su posible relación con $\mathrm{SU}$ y han sido resumidos en cinco metaanálisis.

En tres metaanálisis en los que se evaluó el riesgo de cualquier tipo de cáncer se encontraron resultados contradictorios, en uno se encontró aumento del riesgo en los estudios observacionales prospectivos, pero no se encontró en los ensayos clínicos controlados y en los estudios retrospectivos ${ }^{26}$. Otro metaanálisis no encontró diferencias significativas en los ensayos clínicos controlados y encontró aumento del riesgo en los estudios observacionales prospectivos y retrospecti$\operatorname{vos}^{27}$. Un tercer metaanálisis reportó aumento del riesgo del desarrollo de cáncer, pero no de la mortalidad, sin distinguir los efectos según el tipo de estudio ${ }^{28}$.

Estos metaanálisis declararon varias limitaciones que afectan a la interpretación de sus conclusiones.
En todos la mayoría de los estudios incluidos eran observacionales sin asignación aleatoria, usaban diversos comparadores y dosis y el tiempo de evolución de los pacientes incluidos era muy variable.

En vista de lo anterior consideramos que la evidencia identificada es insuficiente, contradictoria y de baja calidad y no permite afirmar que el tratamiento con SU, como grupo o algún producto en particular, tenga algún efecto sobre el ulterior desarrollo de cáncer.

\section{INDICACIONES EN LA PRÁCTICA CLÍNICA ACTUAL PARA LAS SULFONILUREAS}

Existen evidencias conocidas tomadas de estudios aleatorizados controlados y también de otros de «mundo» o «vida real», multicéntricos y multinacionales, que estiman que el empleo de las SU en las poblaciones con $\mathrm{DM} 2$ es de alrededor del 30-40\%.

Si tomamos dos árboles de decisión sobre tratamiento farmacológico de la DM2 de dos importantes asociaciones médicas (Asociación Americana de Diabetes $[A D A]$ y Colegio Americano de Endocrinólogos Clínicos [AACE]) del mismo año (2018) y del mismo país (EE.UU.) ${ }^{21,29}$, apreciaremos diferencias marcadas referidas a las SU. En el del AACE este grupo, tanto en monoterapia como en terapéutica dual y triple, está ubicado como última opción y además se remarca que las SU deben emplearse con cautela. En el de la $\mathrm{ADA}^{21}$, tanto para terapia doble o triple, en pacientes con $\mathrm{DM}_{2}$ sin eventos cardiovasculares previos (aproximadamente el $70-80 \%$ de los pacientes con DM2 que concurren a los lugares de $1 .{ }^{\text {er }}$ nivel de atención médica, en los estudios de «vida real») se plantea elegir el medicamento individualizándolo de acuerdo con factores referidos al paciente y a efectos específicos del fármaco. 
Refieren como fortalezas de las SU su alta eficacia, su bajo costo y la administración oral. Ubican como dato de interés que el riesgo cardiovascular y en insuficiencia cardíaca es similar a los comparadores. También destacan la neutralidad de las SU en términos de progresión de la nefropatía diabética, y se destaca que la glimepirida y la glipizida deben emplearse con precaución por mayor riesgo hipoglucémico en reducción de filtrado glomerular. Se desaconseja el empleo de glibenclamida (gliburida). Destacan que las SU tienen como grupo la desventaja de aumento de peso corporal y mayor riesgo hipoglucémico. Por lo tanto, a diferencia del AACE, la ADA plantea a las SU como una opción con fortalezas, debilidades y neutralidad cardiovascular y para nefropatía diabética²1.

No existen mayores dudas sobre que este grupo de fármacos tienen una eficacia que genera reducciones de la HbA1c de entre un 1 y un 2\% (mayores cuanto mayor sea la $\mathrm{HbA} 1 \mathrm{c}$ que presenta el paciente y potenciada por asociaciones con otros antidiabéticos). La administración oral es otra ventaja incrementada cuando la misma sea en una dosis diaria.

En cuanto a la neutralidad cardiovascular de las SU, fue demostrada en el mencionado estudio ADVAN$\mathrm{CE}$, donde en cambio, respecto a la supuesta «neutralidad» para nefropatía diabética se constató que la gliclazida reducía un 21\% la aparición o progresión de enfermedad renal diabética en la DM2. Debemos mencionar como cierto el aumento de peso con estos fármacos, pero la magnitud al cabo de los 6 años que duró el seguimiento fue menor a $1 \mathrm{~kg}$. Esto tendría poco impacto clínico, pero es cierto que no tenemos datos en condiciones de «mundo real».

Otra realidad en Latinoamérica es que pese a distintas recomendaciones de evitar glibenclamida, en muchos programas de salud pública en nuestros países se sigue utilizando como un medicamento de gran uso para el tratamiento de la DM2; cuando ello ocurre se debe entregar a los médicos y a los pacientes programas de educación para prevención y tratamiento de las situaciones hipoglucémicas junto a los ineludibles programas de cambios de estilo de vida, que entre muchas otras ventajas limitarán el aumento de peso.

En fin, consideramos que las SU tienen por su eficacia y gran accesibilidad un lugar terapéutico destacado. Este se potencia con aquellas de menor impacto en riesgo de hipoglucemias y de aumento de peso, sobre todo en aquellas moléculas donde hay evidencias fuertes sobre neutralidad en impacto cardiovascular y de beneficio en nefropatía diabética.

\section{LAS SULFONILUREAS EN LAS GUÍAS DE TRATAMIENTO DE DIABETES}

Las SU son medicamentos presentes en todas las guías internacionales de tratamiento de diabetes.

Al ser uno de los grupos farmacológicos más utilizados debe considerarse y conocerse dentro de las opciones terapéuticas para manejar y lograr las metas de control.

En forma amplia se menciona el uso de SU, aunque muchas de las denominadas de primera generación ya se utilizan muy poco en la práctica cotidiana (tolbutamida, clorpropamida y tolazamida). Así mismo, cuando se mencionan las SU estamos analizando las de más reciente generación.

En forma general se las conoce como medicamentos potentes y han sido el marco de referencia de no inferioridad de las moléculas más recientemente introducidas al uso terapéutico. Entre sus efectos clínicos que requieren una vigilancia para su mejor utilización están la mayor frecuencia de hipoglucemia y el aumento leve de peso. 
En las guías de tratamiento están como medicamentos de primera línea para adicionar a la metformina por todos sus beneficios bien reconocidos. En América Latina son fármacos de uso frecuente tanto para pacientes que no toleran la metformina como cuando es necesario armar una combinación con metformina a dosis fijas. La disponibilidad y el costo son los factores decisorios.

Las SU están disponibles para administrarse en conjunto de otro fármaco oral y sus dosis individuales se pueden ajustar buscando lograr metas de control. También se encuentran como dosis fijas con metformina para administrar en una o varias tomas de medicamento. Una de las ventajas que mantiene vigente a las SU es su bajo costo y potencia. Se pueden incluir para combinación de tres fármacos cuando no se logren metas con dos medicamentos y cuando la adición de un mecanismo de acción complementario sea visualizada por el clínico como de beneficio potencial en su utilización ${ }^{21,29,30}$.

Se han analizado las ventajas de la administración de SU y las precauciones necesarias en pacientes de riesgo para hipoglucemias, nefropatía grave, adulto mayor frágil o alguna restricción en su tolerancia.

Como conclusión podemos afirmar que las SU son un grupo farmacológico que sigue vigente en la clínica para el buen control del paciente con DM2 por sus beneficios bien identificados, aunque deben tenerse en cuenta sus limitaciones, hacer los ajustes y cuidados necesarios para evitar riesgos, particularmente hipoglucemia.

\section{BIBLIOGRAFÍA}

1. Loubatières A. [The discovery of hypoglycemic sulfonamides and particularly of their action mechanism]. Acta Diabetol Lat 1969; 6 Suppl 1: 20-56.
2. Khunti K, Chatterjee S, Gerstein HC, Zoungas S, Davies MJ. Do sulphonylureas still have a place in clinical practice? Lancet Diabetes Endocrinol. 2018;6:821-32.

3. King P, Peacock I, Donnelly R. The UK prospective diabetes study (UKPDS): clinical and therapeutic implications for type 2 diabetes. $\mathrm{Br}$ J Clin Pharmacol. 1999;48:643-8.

4. Turner RC. The U.K. Prospective Diabetes Study. A review. Diabetes Care. 1998;21(Suppl 3):C35-8.

5. Malmberg K, Rydén L, Wedel H, Birkeland K, Bootsma A, Dickstein K, et al.; DIGAMI 2 Investigators. Intense metabolic control by means of insulin in patients with diabetes mellitus and acute myocardial infarction (DIGAMI 2): effects on mortality and morbidity. Eur Heart J. 2005;26:650-61.

6. ADVANCE Collaborative Group, Patel A, MacMahon S, Chalmers J, Neal B, Billot L, Woodward M, et al. Intensive blood glucose control and vascular outcomes in patients with type 2 diabetes. $\mathrm{N}$ Engl $\mathrm{J}$ Med. 2008;358:2560-72.

7. Viberti G, Kahn SE, Greene DA, et al. A diabetes outcome progression trial (ADOPT): an international multicenter study of the comparative efficacy of rosiglitazone, glyburide, and metformin in recently diagnosed type 2 diabetes. Diabetes Care 2002; 25: 1737-43.

8. Kahn SE, Zinman B, Lachin JM, Herman WH, Zinman B, Holman RR, et al. Rosiglitazone-Associated Fractures in Type 2 Diabetes: An analysis from A Diabetes Outcome Progression Trial (ADOPT). Diabetes Care. 2008;31:845-51.

9. Gæde P, Lund-Andersen H, Parving H-H, Pedersen O. Effect of a multifactorial intervention on mortality in type 2 diabetes. N Engl J Med. 2008;358:580-91.

10. Kalra S, Bahendeka S, Sahay R, Ghosh S, Md F, Orabi A, et al. Consensus Recommendations on Sulfonylurea and Sulfonylurea Combinations in the Management of Type 2 Diabetes Mellitus - International Task Force. Indian J Endocrinol Metab. 2018;22:132-57.

11. Colagiuri S, Matthews D, Leiter LA, Chan SP, Sesti G, Marre M. The place of gliclazide MR in the evolving type 2 diabetes landscape: $\mathrm{A}$ comparison with other sulfonylureas and newer oral antihyperglycemic agents. Diabetes Res Clin Pract. 2018;143:1-14.

12. Powers A, D'Alessio D. Páncreas endocrino y farmacoterapia de la diabetes mellitus e hipoglucemia. En: Brunton LL, Chabner BA, Knollmann BC, editores. Goodman \& Gilman. Las bases farmacológicas de la terapéutica, 12. ${ }^{a}$ ed. México DF: McGraw-Hill; 2012. pp. 1237-73.

13. Nathan DM, Buse JB, Kahn SE, Krause-Steinrauf H, Larkin ME, Staten $M$, et al.; GRADE Study Research Group. Rationale and design of the glycemia reduction approaches in diabetes: A comparative effectiveness study (GRADE). Diabetes Care. 2013;36:2254-61.

14. Kahn SE, Haffner SM, Heise MA, Herman WH, Holman RR, Jones NP, et al.; ADOPT Study Group. Glycemic durability of rosiglitazone, metformin, or glyburide monotherapy. N Engl J Med. 2006;355: 2427-43.

15. Wong MG, Perkovic V, Chalmers J Woodward M, Li Q, et al. Long-term benefits of intensive glucose control for preventing end-stage kidney disease: ADVANCE-ON. Diabetes Care. 2016;39:694-700.

16. Marx N, Rosenstock J, Kahn SE, Zinman B, Kastelein J, et al. Design and baseline characteristics of the CARdiovascular Outcome Trial of LINAgliptin Versus Glimepiride in Type 2 Diabetes (CAROLINA ${ }^{\circledR}$ ). Diabetes Vasc Dis Res. 2015;12:164-74.

17. Neil WP, Hemmen TM. Neurologic manifestations of hypoglycemia. En: Rigobelo E, editor. Diabetes - Damages and Treatments. InTech; 2011. pp. 259-74.

18. Douros A, Yin H, Yu OHY, Filion KB, Azoulay L, Suissa S. Pharmacologic differences of sulfonylureas and the risk of adverse cardiovascular and hypoglycemic events. Diabetes Care. 2017;40:1506-13.

19. Van Dalem J, Brouwers M, Stehouwer C, Krings A, Leufkens H, et al. Risk of hypoglycaemia in users of sulphonylureas compared with 
metformin in relation to renal function and sulphonylurea metabolite group: population based cohort study. BMJ. 2016; 354: i3625.

20. Leonard C, Bilker W, Brensinger C, Han X, Flory J, et al. Severe hypoglycemia in users of sulfonylurea antidiabetic agents and antihyperlipidemics. Clin Pharmacol Ther. 2016;99:538-47.

21. Standards of Medical Care in Diabetes-2018. Diabetes Care. 2018; 41(Suppl 1)S1-S159.

22. Pop LM, Lingvay I. The infamous, famous sulfonylureas and cardiovascular safety: Much ado about nothing? Curr Diab Rep. 2017;17:124.

23. Rados DV, Pinto LC, Remonti LR, Leitão CB, Gross JL. Correction: The association between sulfonylurea use and all-cause and cardiovascular mortality: A meta-analysis with trial sequential analysis of randomized clinical trials. PLOS Med. 2016;13:e1002091.

24. Anagnostis P, Siolos P, Christou K, Gkekas N, Kosmidou N, et al. The effect of antidiabetic medications on the cardiovascular system: a critical appraisal of current data. Hormones. 2018;17:83-95.

25. Hemmingsen B, Schroll J, Lund S, Wetterslev J, Gluud C, et al. Sulphonylurea monotherapy for patients with type 2 diabetes mellitus. En: Hemmingsen B, editor. Cochrane Database of Systematic Reviews. Chichester, UK: John Wiley \& Sons; 2013.
26. Thakkar B, Aronis KN, Vamvini MT, Shields K, Mantzoros CS. Metformin and sulfonylureas in relation to cancer risk in type ii diabetes patients: A meta-analysis using primary data of published studies. Metabolism. 2013;62:922-34.

27. Chen Y, Wu F, Saito E, Lin Y, Song M, et al. Association between type 2 diabetes and risk of cancer mortality: a pooled analysis of over 771,000 individuals in the Asia Cohort Consortium. Diabetologia. 2017; 60:1022-32.

28. Wu L, Zhu J, Prokop LJ, Murad MH. Pharmacologic therapy of diabetes and overall cancer risk and mortality: A meta-analysis of 265 studies. Sci Rep. 2015;5:10147.

29. Garber AJ, Abrahamson MJ, Barzilay JI, Blonde L, Bloomgarden Z, et al. Consensus Statement by the American Association of Clinical Endocrinologists and American College of Endocrinology on the Comprehensive Type 2 Diabetes Management Algorithm - 2018. Endocr Pract. 2018;24:91-120.

30. McGuire H, Longson D, Adler A, Farmer A, Lewin I; Guideline Development Group. Management of type 2 diabetes in adults: summary of updated NICE guidance. BMJ. 2016;353:11575. 